\title{
Citation for a discussion of noncompliance
}

Dr. Larry Covelli was proud of the IACUC he chaired at Great Eastern University. There was a culture of thorough but reasonable protocol reviews, open discussions at meetings and an honest effort to balance animal welfare with research requirements. Why, then, was he morose and bitter after talking to the IACUC Administrator?

Unknown to the IACUC, an investigator without an IACUC protocol was given a single-needle biopsy sample taken from the liver of a sheep that was being used in a survival abdominal surgical procedure for an unrelated purpose. The sheep study did have IACUC approval, but the approval did not include the biopsy. The Principal Investigator (PI) of the sheep study thought he was just doing a favor for a colleague who needed a small sample of liver tissue. In fact, as was later learned, the PI thought he was reducing animal usage because his colleague would not have to use an entirely separate animal for the biopsy. As for the sheep, she recovered uneventfully.
All of this occurred on a Monday, and by the next morning, Covelli knew of the incident. He quickly contacted both investigators, told them why they were noncompliant with NIH/OLAW policy and USDA/Animal Care regulations, and stated that no further biopsies were to be done without an approved protocol amendment or a new protocol. Coincidentally, the monthly IACUC meeting took place that Wednesday, and at the meeting, Covelli dutifully reported what had transpired. After a discussion, the committee concluded that the actions taken by Covelli were appropriate and voted to send a letter of explanation and reprimand to both investigators. Although the incident was a protocol noncompliance, the discussion and vote indicated it was not serious enough to inform NIH/OLAW of the incident and its resolution.

Some months later, during a routine USDA/Animal Care inspection, the inspecting veterinarian read the minutes of the IACUC meeting during which the sheep incident was discussed. He then cited Great Eastern for inadequate training of research personnel and for not having an appropriate proposal to conduct an animal activity. Covelli was mortified. "My God," he said, "if there was ever a reason to hide animal welfare problems under the rug, this is it. There was no requirement for us to report anything to the USDA but we got slammed nevertheless for just discussing it. And now it's going to be publically posted and blown out of proportion. Why not just cite us for every repair order that's submitted or every time the vets ask a PI to change an anesthetic dosage. This is ridiculous and untenable!"

Now we know why Covelli was morose and bitter. But did he have a reasonable complaint, or was he just angry that Great Eastern received an IACUC-focused USDA citation while under his watch? Do you think Covelli was right when he implied that less will be said at future IACUC meetings about protocol noncompliance with USDA-covered species?

\section{RESPONSE}

\section{Don't hide it}

Patricia N. Coan, DVM, PhD, DACLAM, William A. Hill, DVM, MPH, DACLAM, CPIA \& Joleen K. Adams, DVM

Although the investigators thought they were being mindful in reducing animal numbers, the fact remains that animal procedures were carried out without IACUC approval.

Should the USDA have been notified? The institution was not required to report to the USDA, as the IACUC did not suspend the protocol (9 CFR 2.31.d.7) ${ }^{1}$. According to the PHS Policy on Humane Care and Use of Laboratory Animals (IV.F.3.a) ${ }^{2}$, however, OLAW could have been notified: "The IACUC, through the Institutional Official, shall promptly provide OLAW with a full explanation of the circumstances and actions taken with respect to: any serious or continuing noncompliance with this Policy." Could the institution report a noncompliant event to OLAW? Yes; carrying out animal procedures without IACUC approval is a serious noncompliance. This can be considered a reportable offense, depending on the institution's IACUC procedures and policies.

Should the USDA cite noncompliant incidents that have already been identified and resolved? The liver biopsy was not a part of the original protocol, and so it was carried out without IACUC approval. The USDA can cite noncompliant incidents that happened previously. Perhaps the USDA did not think that the institution took appropriate corrective action by just sending a letter of reprimand to the investigators. Was additional training required? What procedures did the IACUC put into place to prevent future occurrences? Was the current investigator training program adequate? Was there post-approval monitoring in place? Did the minutes reflect the discussion of the IACUC regarding compliance issues?

We understand Covelli's frustration at being cited for something that he thought the IACUC had discovered and handled. The citation was for the noncompliant event, not for the IACUC's response. The investigators who were involved in the noncompliance obviously needed more training. Covelli must realize that there are many different viewpoints and interpretations by IACUC members and regulatory personnel.

Was Covelli right when he implied that less would be said about noncompliance? 